\title{
IMAGE DENOISING: A COMPARATIVE STUDY OF VARIOUS WAVELET APPROACHES
}

\author{
Laavanya Mohan ${ }^{*}$ and Vijayaragahvan Veeramani ${ }^{1}$ \\ Vignan's Foundation for Science Technology and Research, \\ Vadlamudi, Guntur, Andhra Pradesh - 522213, India. \\ laavanvijay@gmail.com,vijayaragahvan123@gmail.com
}

\begin{abstract}
:
Image denoising is a major tricky in image processing. The main determination is to quash noise from the degraded image while keeping other details of the image unchanged. In recent years, many multiresolution based approaches have attained great success in image denoising. In a nut shell, the wavelet transform provide an optimal representation of a noisy image, including a signal with information from a limited number of coefficients and noise by all other left over coefficients. The most popular way to eliminate noise is to threshold the noise affected wavelet coefficient. The noise affected wavelet coefficient shrinkage is better, only if the threshold value is properly selected. Therefore, the performance of various wavelet based denoising techniques depends on the estimation of the threshold value. Different techniques are available to find the threshold value. The aim of this study is to discuss denoising schemes based on various wavelet transforms using threshold approach. Hence, this article examines the research article with threshold selection based on spatial adaptivity, sub-band adaptivity and also hybrid methods with multi-resolution wavelet structures.
\end{abstract}

Keywords: Image denoising, Image processing, Thresholding function, Wavelet transform.

\section{INTRODUCTION}

Digital photography plays an important role in everyday life. This leads to the need for image manipulation. The three stages of image manipulation are, image processing, image analysis and image comprehension. In image processing input and output is an image. In image analysis the input is an image and the result is an image measurement. In image comprehension the input is an image and also output a high definition image.

Image processing can be defined as enhancing the visual appearance of images (or) extracting information contained in an image. Often, images are corrupted by noise during detection, transmission and retrieval process. Digital images are prone to a variety of noises such as Gaussian noise, salt and pepper noise, speckle noise, shot noise and many more. Depending on how the noise affects the image, noise can be classified as additive and repetitive sounds. A dditive noise is considered here, because at the time of image acquisition the color image is converted into a continuous electronic signal used for sampling to obtain a digital image. At every process the natural fluctuations adds a random value to the exact brightness value of the pixel and it is given by

$$
w(x, y)=s(x, y)+n(x, y)
$$

Where $\mathrm{s}(\mathrm{x}, \mathrm{y})$ is the original image, $\mathrm{n}(\mathrm{x}, \mathrm{y})$ is the noise and $w(x, y)$ is the noise contaminated image, here $(\mathrm{x}, \mathrm{y})$ means the pixel location. This type of noise is called as Additive White Gaussian Noise (AWGN). Gaussian sound has the potential for a workload equal to that of standard distribution. The probability density function $\mathrm{p}$ of a Gaussian random variable $\mathrm{z}$ is given by

$$
P G(z)=\frac{1}{\sigma \sqrt{2 \pi}} e^{-\frac{(z-\mu)^{2}}{2 \sigma^{2}}}
$$

In equation (2) $\mathrm{z}$ represents the grey matter, $\mu$ is the mean value and $\sigma$ is the standard deviation. In image denoising there is a correlation between noise reduction and conservancy of image particulars. Discrete wavelet change is an excellent tool in image processing was addressed by Mallat (1989). Wavelet transform harvests a reckoning set of coefficients in the transform domain. Therefore, most of the signal strength is contained in large coefficients and a small portion of the energy is distributed by a large number of small coefficients. It has properties like sparseness, clustering and correlation between neighbouring wavelet coefficient. Another variation of wavelet transform is the real oriented dual tree discrete wavelet transform. This can be done using two real separable 2D wavelet transform in parallel. This is a directional orientation transform and can be implemented by addition and subtraction of individual sub-bands of two 2D detachable 
real wavelet transform. The transform is free of checker board artifact, but wavelet coefficients are not complex and hence it has not approximately shifted invariance. The details like edges and contours play more roles in visual perception. An efficient tool for preventing all these image details is the DTCWT was introduced by Kingsbury (1998). DTCWT is a computationally competent methodology to shift invariance and has better directional selectivity. DTCWT uses separable filter banks as discrete wavelet transform, but it is superior, since the magnitude and phase of complex wavelet transform can be used to develop new effective wavelet based algorithms where DWT can't be used.

This has motivated to study denoising algorithms based on various wavelet transforms. Hence, in the present study four different schemes for Gaussian noise reduction, with efficacious approach for threshold selection by conserving all other features of image is discussed in detail.

\section{RELATED WORKS:}

To retrieve the noise free image, denoising is needed. The purpose of denoising technique is to remove noise by conserving all other features of image. In spatial and frequency domain several approaches for noise reduction are proposed.

The spatial domain methods are further classified into linear and nonlinear methods. A linear filter removes the noise by decreasing the intensity value between the neighbouring pixels by using a mask on every pixel was proposed by (Ozkan et al., 1992). Median stack filter and generalized median filter are all linear filters that fail to preserve the edges were addressed by (Gabbouj et al., 1992) and (Lee and Kassam, 1985). Edge preserving spatial filters like SUSAN filter proposed by (Smith and Brady, 1997) is based on the intensity and distance is calculated as a measure of correlation between pixels. The disadvantage of SUSAN filter is that they depend only on intensity and not on spatial locality. This was overcome by (Tomasi and Manduchi, 1998) who projected a bilateral filter based on both intensity and spatial distance between the pixels. The most important tricky with bilateral filter is the parameter choice and the filter perform well to preserve edge and fails when the noise level is high. This was addressed by (Zhang and Gunturk, 2008) as an empirical study of noise variance and also explained the application of the bilateral filter in wavelet domain. But in wavelet domain the bilateral filter flattens the gray level and results in a cartoon like appearance of the denoised image. This drawback can be overcome by using a combination of Gaussian/bilateral filter proposed by (Shreyamshakumar, 2013).

The non-linear methods, transform the image as smoothly varying regions with piecewise constant regions are addressed by (Acton and Bovik, 1998; Gabbouj et al., 1992; Lee and Kassam 1985). The nonlinear technique called SVD, can be successfully used to denoise an image was introduced by (Andrews and Patterson, 1976). In this method, SVD is applied to the complete image in a solitary step, but it lacks in distinguishing significant and non-significant singular values, and edges are preserved only to a certain extent. In order to reserve the edge partial differential equation based approaches, use an edge detection term to flat the edge structure was proposed by (Arce and Foster, 1989; Smith and Brady, 1997). (Tomasi and Manduchi, 1998) established a denoising approach with edge preservation that independently enforces geometric closeness and grey value from the wellknown Bayesian approach.

Among transform domain methods wavelet transform is considered to be a successful approach for image denoising, due to its vitality dense property introduced by (Mallat, 1989; Raghuveer and Bopardikar. 1999; Mohideen et al., 2008). The wavelet has the ability of de-correlation of the dissimilar discrete wavelet transforms. This is for the reason that, the information is comprising in small number and all other coefficients contain noise. (Donoho, 1995; Coifman and Donoho, 1995) proposed a denoising method to filter these noisy coefficients using a thresholding scheme. Several threshold selection strategies like VisuShrink proposed by (Donoho and Johnstone, 1994) SureShrink proposed by (Donoho et al., 1995) and BayesShrink proposed by (Chang et al., 2000), produce denoised image based on the choice of the threshold value. (Chen et al., 2004) projected a thresholding structure by incorporating neighbouring coefficients named as NeighShrink. This method uses only soft thresholding approach and does not take into account about multi wavelet coefficients. This disadvantage was diminished by using a pre-filter, multi wavelet transform, thresholding scheme by neighbouring multi wavelet coefficients and finally taking the inverse followed by post filtering was proposed by (Chen and Bui, 2003). But this scheme has not considered the bigger neighbourhoods, just immediate neighbours are considered. This problem was addressed by (Dengwen and Wengang, 2008) where an best threshold and neighbouring window size is resolute for every sub-band using SURE. (Cho et al., 2009) proposed NeighLevel shrinkage where the parent wavelet coefficients and the neighbours are created on NeighShrink tactic harvests fairly sustaining results. The denoising result can be 
improved if a better adaptive threshold is used. The threshold value is determined for every level by identifying the coefficients of a neighbouring window for every sub-band was proposed by (Hari and Mantosh, 2012; 2015).

Further the enslavements flanked by a coefficient and its parent are measured and the equivalent bivariate shrinkage function is imitative using Bayesian estimation theory was presented by (Sendur and Selesnick, 2002a; 2002b). By means of local adaptive methods in amalgamation with bivariate shrinkage may promote the denoising enactment. Shrinkage is better when parent coefficient is smaller. Bivariate function can also be used with other transform domain approaches to denoise an image was proposed by (Zhang et al., 2013).

While all methods are easy to use, the reconstruction of a noise affected image suffers from the transition to movement and directional selectivity. This problem be located by (Kingsbury, 1998; 1999; 2001; Selesnick et al., 2005) using a dual tree filter to obtain real and imaginary parts of complex coefficients. The two-wave filter announces a 4: 1 reduction in $2 \mathrm{D}$ signals. The transform therefore produces the flexibility of movement and directional selectivity.

There are two versions of DTDWT: the first is the actual version of 2D-DTDWT and the next is the complex 2D-DTDWT. The Daubechies wavelet converter is proposed by (Khare et al., 2010), with the flexible limit and shrinkage function used to perform denoising, resulting in better performance when the noise level is high. But the approach is not pleasant for all small levels of noise. (Chen et al., 2012) successfully applied the complexity of a complex tree transformation into a sound image by paying attention to the wavelet coefficients on three scales during the thresholding process. The denoising performance can still be improved if the statistical dependence between wavelet coefficients is observed. Recently (Remenyi et al., 2014) proposed the modification of the complex 2D mixing process by establishing a covariance structure using a powerful Bayesian process that results in better denoising performance. The method proposed by (Naveed et al., 2019) uses localized GoF experiments with noise image relationships derived from the discrete wavelet transform (DWT) and the complex two-tree wavelet transform (DT-CWT). The image structure is constructed as a binary hypothesis test with a null hypothesis that indicates the presence of sound and another hypothesis that represents the presence of only the desired signal. Determination of a wave coefficient similar to the null hypothesis or other method includes the GoF test based on the empirical distribution function (EDF), which is applied locally to the noise factors, provides good noise reduction characteristics. The method proposed by (Lavanya et al., 2020) uses two BR phases. The first section predicts the type of image, while the second section estimates the corresponding wave coefficients in the DT-CWT by subtracting the noise. The effectiveness of this proposed model is assessed based on the signal-to-noise ratio, the second measure of enhanced gain, structural similarity, standard error, standard error, error mean absolute, Pearson's coefficient and symmetric mean absolute error in percentage, respectively. The proposed model works best in terms of noise reduction.

Unraveling the method using DTCWT, using suitable wave filters for design and use in FPGA, detailed analysis was done in Matlab Simulink R2012b software using db9, sym9, airframe4, bior6.8 and Farras Long-10 wave filters for 2D sofas - The degree of DTCWT based on images created by soft divination using the Vinero's filter method and simulation software design was created with Xilinx ISE 14.5 and has taken effect in the Spartan 3E FPGA kit. The results show that the structure consumes a total power of $300137 \mathrm{~mW}$ and operates at a frequency of 173340 MHz, addressed by (Faruk et al., 2017).

Both spatial and transform domain based approaches have achieved a great success in image denoising. The hybrid of both domains has also given a better denoising performance. The well-known spatial domain method SVD can be used with transforming domain methods like: SVD is applied to the discrete cosine transformed image for denoising was proposed by (Wongsawat et al., 2005). In this approach time consumption is high and small amount of high frequency content is retained with the original image. SVD has also been proposed as a multiresolution form of filter for signal analysis by (Kakarala and Ogunbona, 2001). In multi-resolution analysis SVD packs the energy of the signal into as few coefficients as possible. Hence SVD has the ability to manipulate the noisy image into data and noise subspaces was proposed by (Zujun, 2003).

The authors (Iqbal et al., 2014) propose the conversion of a complex wave into a double tree and the decomposition of the single value to improve the resolution of medical images using a non-local media filter. Quantitative and qualitative analysis is used to find the effectiveness of the proposed technique. The SVD and DTCWT hybrid is used to reduce noise and detect pipeline leaks, was suggested by (Yu et al., 2016). In this document, DTCWT is a deliberate core for achieving multi-phase decomposition and 
refinement, for audio signals, and uses SVD to eliminate noise in non-typical bands.

Based on the above study, it is apparent that every denoising procedure has the same objective, which is to obtain a noise free image by mathematically minimizing the fault flanked by the original and recovered images. The survey outcomes demonstrate that hybrid methods with sparse representation, spatial and sub-band adaptivity perform better than existing denoising techniques. Hence, in this paper four denoising approaches presented by (Laavanya and Karthikeyan, 2016; 2017, 2018; 2019) based on the above survey results is studied and compared.

\section{COMBINATION OF SPATIAL AND TRANSFORM DOMAIN APPROACHES FOR IMAGE DENOISING APPLICATION}

\subsection{SVD and Bivariate Model in Wavelet Domain}

Here Singular Value Decomposition (SVD) with the bivariate shrinkage function for image denoising in wavelet domain is presented. It is noteworthy as compared to the similar schemes in the wavelet domain; this simple scheme is much better in terms of PSNR values and chromatic worth.

This scheme is evidently not superior to the latest denoising schemes. However, in this method the concept of SVD is used in the transformed image and the singular values are approximated instead of truncated. The threshold value is computed using noise and signal variance which is locally adaptive is introduced.

This approach has four steps, first to transform the input noisy image, secondly to perform SVD and Frobenius energy correction for the transformed wavelet coefficients, then threshold the coefficients and finally reconstruct the image using modified coefficients.

\subsection{Real Oriented DTWT with an Optimal Threshold using Neighbor Coefficients and Gaussian Bilateral Filter}

As compared to previous method three major modifications are made one is the use of real oriented 2D Dual Tree Wavelet Transform (DTWT), the second one is a added effective way of identification of optimal threshold using neighbour coefficient based on SURE risk, and the third one is to use of Gaussian / bilateral filter to reserve the edges/ sharp borders in the image.

The truly oriented 2D DTWT used here is an extension of the DWT-based noise reduction technique. The truly oriented 2D DTWT is also referred to as inseparable wavelets. This wavelet can be realized using two truly separable $2 \mathrm{D}$ wave transformations. By applying the two separate waveform transformations to the same 2D data, a total of six sub-bands, two LH, two HL and two HH subbands are obtained. The transformation is performed by taking the sum and difference of each pair of subbands. This results in a transformation that is twice that of DWT and without checkerboard artifacts.

Here two schemes are discussed in the first one optimal threshold is estimated by considering the whole noisy image with constant window size using NeighSURE. Then the noisy image undergoes real oriented 2D DTWT up to $\mathrm{j}$ scales resulting in a sparse depiction of the image. The optimal threshold is applied to the entire sub-bands using soft thresholding. Finally the reconstructed denoised image from modified wavelet coefficients undergoes post filtering using a Gaussian / bilateral filter to preserve the edge structures. In the second scheme same procedures are used as above, except in finding the optimal threshold value. The best threshold is computed by varying the window size in accordance to the changes in the noise variance.

\subsection{DTCWT using Sub-Band Adaptive Visushrink}

In this section a sub-band adaptive Visushrink based denoising scheme is presented using Dual Tree Complex Wavelet Transform (DTCWT). This scheme is different from the earlier two schemes in two major aspects. The first one is the estimation of sub-band adaptive signal variance. The second one is a sub-band adaptive threshold.

The Dual Tree wavelet is a revolutionary solution with multiple variants, image representation. It has a transfer switch and directional selection as well as a redundancy factor of $2 \mathrm{~d}$ size feature compared to DWT. DTCWT combines complex coefficients using two different DWTs. The first real DWT provides the real part of the wavelet coefficients and the second provides the imaginary portion of the wavelet coefficients. In DTCWT the real and imaginary parts do not change by purging alone. Here is a denoising method in which signal variations are detected on all scales, using a neighboring window. The VisuShrink sub-band flexible limit is then calculated from the converted coefficients and applied to all bands. Finally the reconstructed image is reconstructed from the coelfficients under the adjusted band. This approach is truly simple and works well with more and more choice of direction and movement change 


\subsection{DTCWT Incorporating SVD and Bilateral Filter}

In this section 2D dual tree complex wavelet transform is used for image denoising technique because, Dual Tree Complex Wavelet Transform (DTCWT) can give a significant recital boost to DWT noise reduction techniques. Hence this scheme is different from the scheme presented in the above chapters in two aspects. One is the use of DTCWT to get a better sparse representation of the image. Second change is the estimation of new low pass sub-band using SVD and Frobenius energy correcting factor and finally bilateral filter for smoothing.

The image to be denoised is first transformed by DTCWT on six scales. SVD is then applied to the low pass sub-band. The new low pass sub-band coefficients are computed by using Frobenius energy correcting factor followed by bilateral filtering. The noise free wavelet coefficients are estimated by a local adaptive bivariate function.

With the help of bivariate, the heavy tail behaviour of natural images is accurately modeled and the inter-scale properties of the wave coefficients are properly used. Finally by taking inverse DTCWT the noise free image is obtained.

\section{COMPARISION OF DENOISING METHODS IN MULTIRESOLUTION REPRESENTATION}

In this section the above discussed four methods are compared both visually and numerically. A numerical measure of image quality is PSNR. Here grey scale images are used and their range is [0, 255]. For different noise variances, the measured PSNR value for four different methods are listed in table 1 and also shown as graphs in figures 1,2 and 3. The table 1 gives the PSNR values of the four proposed techniques for standard gray scale test images like Lena, Boat and Barbara. Table 1 shows that the fourth method using DTCWT and SVD accomplish an efficacious noise diminution at various noise power levels $\sigma=[10,15$, 20, 25, 30] compared to other three methods. The better performance of DTCWT and SVD is due to two reasons. First the choice of threshold value is adaptive and secondly very small noise in low pass band is suppressed by Frobenius energy correction factor. The evaluation results of Lena, Boat and Barbara images are shown separately in figures 1,2 and 3 respectively. From the figures it is evident that among the four methods DTCWT outperforms other three methods.

Table 1. Comparison of PSNR values of the four denoising methods

\begin{tabular}{|c|c|c|c|c|}
\hline \multirow{3}{*}{ Proposed Methods } & $\boldsymbol{\sigma}_{\mathbf{n}}$ & LENA & BOAT & BARBARA \\
\hline \multirow{3}{*}{ SCHEME_1 } & 10 & 34.31 & 32.35 & 32.14 \\
\cline { 2 - 5 } & 15 & 32.45 & 30.50 & 29.80 \\
\cline { 2 - 5 } & 20 & 31.12 & 29.15 & 28.19 \\
\cline { 2 - 5 } & 25 & 30.12 & 28.08 & 27.01 \\
\hline \multirow{3}{*}{ SCHEME_2 } & 30 & 29.29 & 27.30 & 26.16 \\
\cline { 2 - 5 } & 10 & 34.06 & 31.93 & 30.95 \\
\cline { 2 - 5 } & 15 & 32.25 & 30.20 & 28.92 \\
\cline { 2 - 5 } & 20 & 30.96 & 29.004 & 27.64 \\
\hline \multirow{3}{*}{ SCHEME_3 } & 25 & 29.78 & 27.72 & 26.19 \\
\cline { 2 - 5 } & 10 & 34.97 & 32.43 & 32.84 \\
\cline { 2 - 5 } & 15 & 33.27 & 30.67 & 30.69 \\
\cline { 2 - 5 } & 20 & 30.99 & 29.40 & 29.21 \\
\hline \multirow{3}{*}{ SCHEME_4 } & 25 & 30.17 & 28.41 & 27.06 \\
\cline { 2 - 5 } & 30 & 40.63 & 38.04 & 38.39 \\
\cline { 2 - 5 } & 10 & 38.70 & 35.98 & 34.28 \\
\hline
\end{tabular}




\begin{tabular}{|l|l|l|l|l|}
\hline \multirow{2}{*}{} & 25 & 36.09 & 33.38 & 32.99 \\
\cline { 2 - 5 } & 30 & 35.28 & 32.42 & 31.84 \\
\hline
\end{tabular}

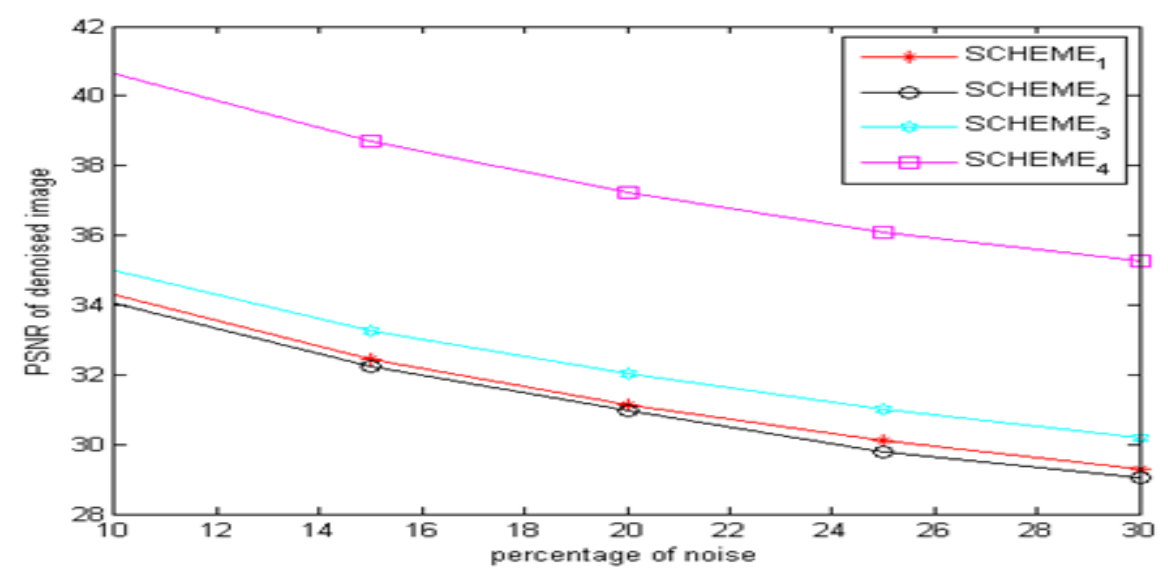

Fig. 1. Comparison of PSNR Value of four denoising methods for Lena Image

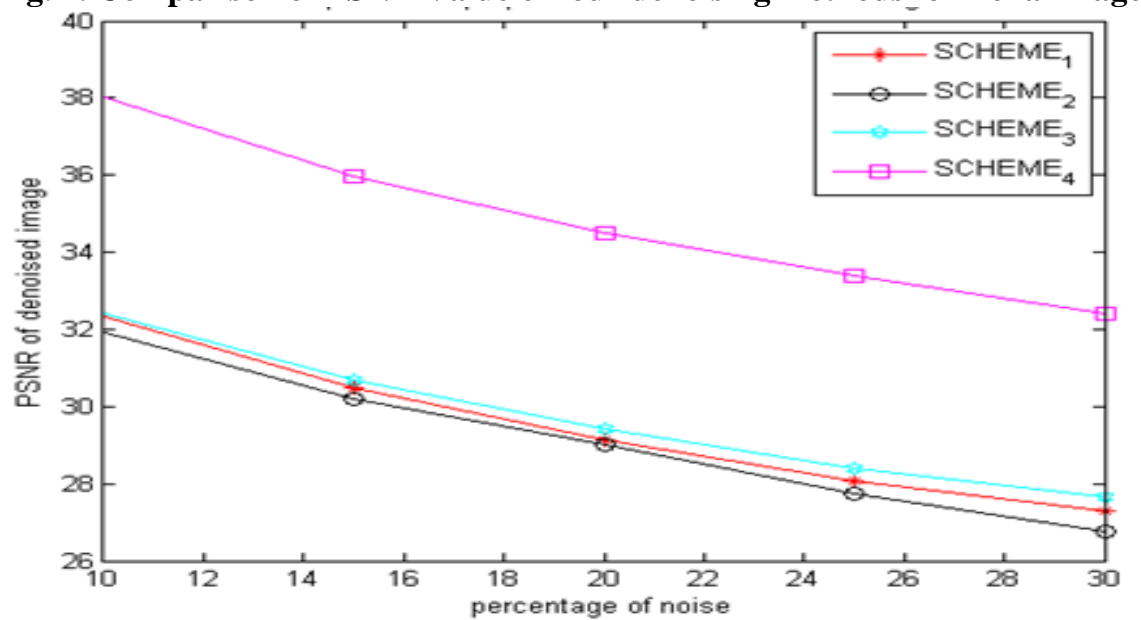

Fig. 2 Comparison of PSNR Value of four denoising methods for Boat Image

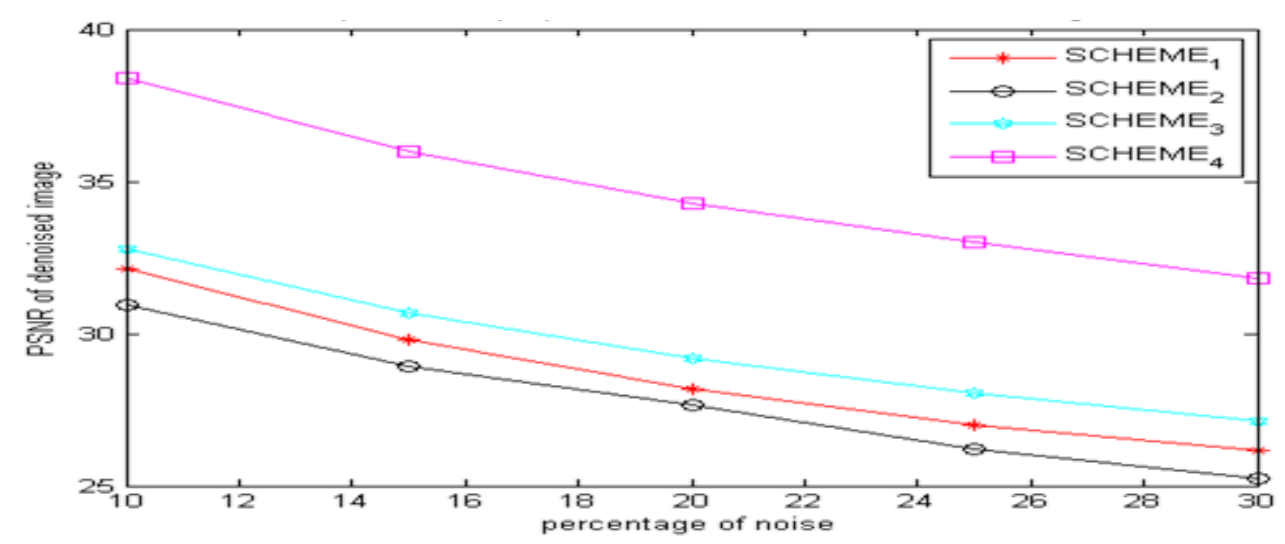

Fig. 3 Comparison of PSNR Value of four denoising methods for Barbara Image

Here MSSIM is used as a visual appearance, performance metrics. SSIM is used to measure the similarity between the original image and denoised image. SSIM gives the distortion as a combined 
measure of three factors: luminance distortion, loss of correlation and contrast distortion. Hence it provides a perceptual image error measure. The table 2 and figures (graphs) 4, 5, and 6 gives the comparison of these parameters for the four methods. From table 2 we can see that SSIM values for DTCWT and SVD based method is better than other three methods. It can also be seen that SSIM values at noise variance 30 is close to one. If SSIM value is close to one, then it indicates noise reduction in the image is better. Figures 4, 5 and 6 shows the performance of images Lena, Boat and Barbara. The improvement in the denoising performance is shown in graphs for various noise variance.

Table 2. Comparison of SSIM values of the four denoising methods

\begin{tabular}{|c|c|c|c|c|}
\hline $\begin{array}{l}\text { Proposed } \\
\text { Methods }\end{array}$ & $\sigma_{\mathbf{n}}$ & LENA & BOAT & BARBARA \\
\hline \multirow{5}{*}{ SCHEME_1 } & 10 & 0.8897 & 0.8524 & 0.9031 \\
\hline & 15 & 0.8581 & 0.8092 & 0.8598 \\
\hline & 20 & 0.8327 & 0.7686 & 0.8177 \\
\hline & 25 & 0.8096 & 0.7360 & 0.7826 \\
\hline & 30 & 0.7916 & 0.7116 & 0.7491 \\
\hline \multirow{5}{*}{ SCHEME_2 } & 10 & 0.8945 & 0.8508 & 0.8900 \\
\hline & 15 & 0.8636 & 0.8109 & 0.8469 \\
\hline & 20 & 0.8255 & 0.7724 & 0.8056 \\
\hline & 25 & 0.8027 & 0.7327 & 0.7584 \\
\hline & 30 & 0.7783 & 0.6981 & 0.7198 \\
\hline \multirow{5}{*}{ SCHEME_3 } & 10 & 0.9005 & 0.8504 & 0.9120 \\
\hline & 15 & 0.8773 & 0.8105 & 0.8779 \\
\hline & 20 & 0.8551 & 0.7770 & 0.8462 \\
\hline & 25 & 0.8358 & 0.7484 & 0.8145 \\
\hline & 30 & 0.8203 & 0.7230 & 0.7856 \\
\hline \multirow{5}{*}{ SCHEME_4 } & 10 & 0.9049 & 0.8639 & 0.9193 \\
\hline & 15 & 0.8815 & 0.8294 & 0.8890 \\
\hline & 20 & 0.8621 & 0.7980 & 0.8602 \\
\hline & 25 & 0.8421 & 0.7688 & 0.8318 \\
\hline & 30 & 0.8254 & 0.7440 & 0.8049 \\
\hline
\end{tabular}

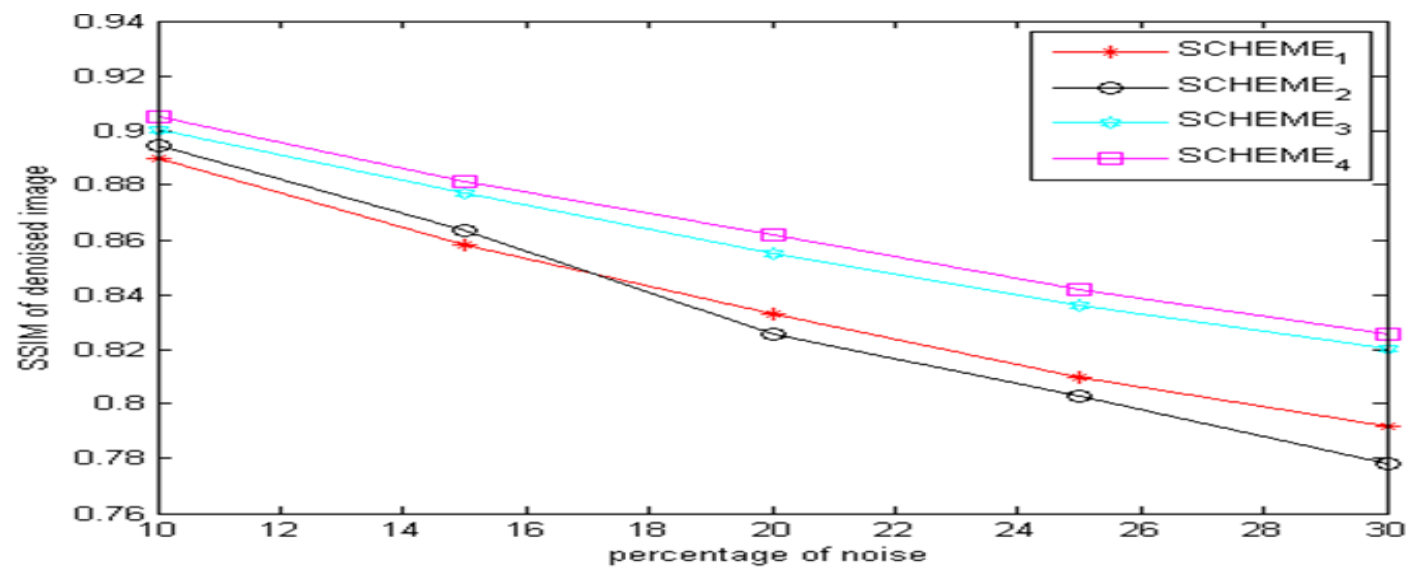

Fig. 4 Comparison of MSSIM Value of four denoising methods for Lena Image 


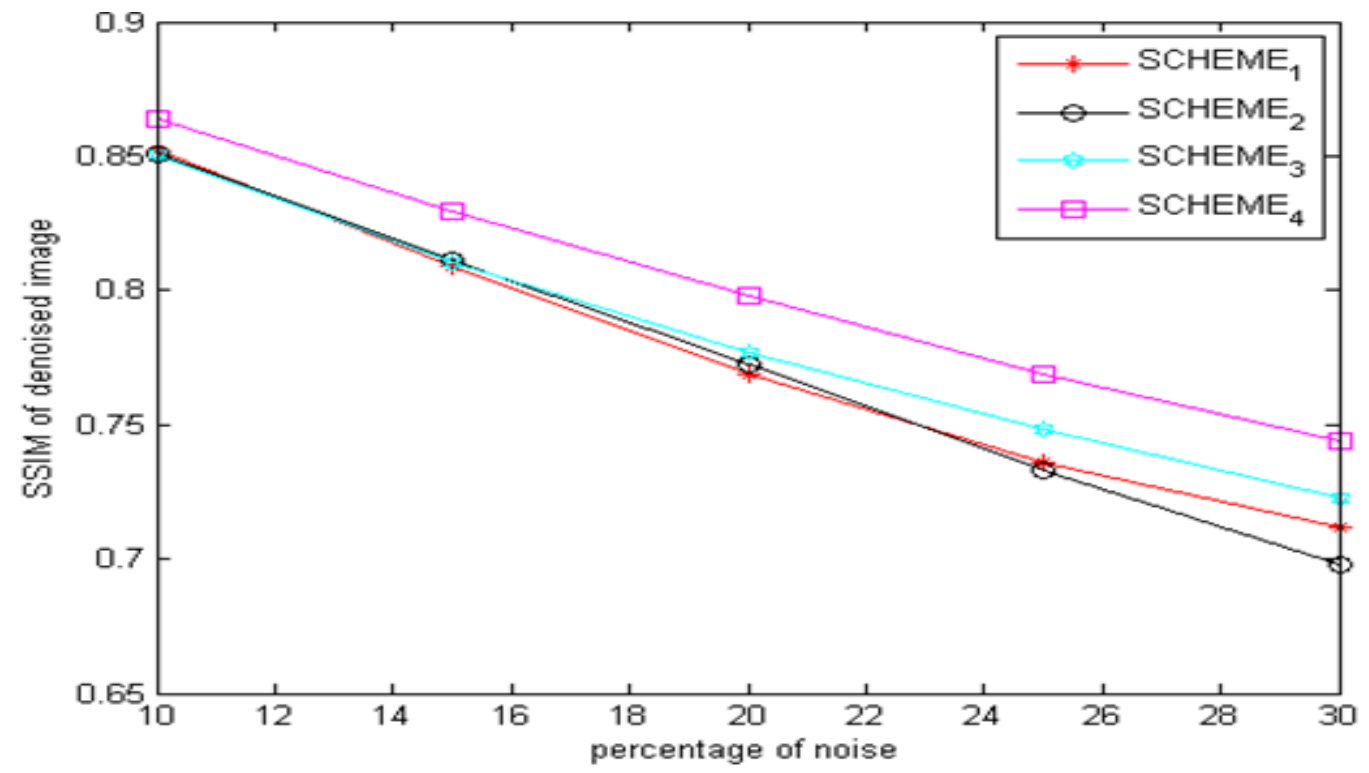

Fig. 5 Comparison of MSSIM Value of four denoising methods for Boat Image

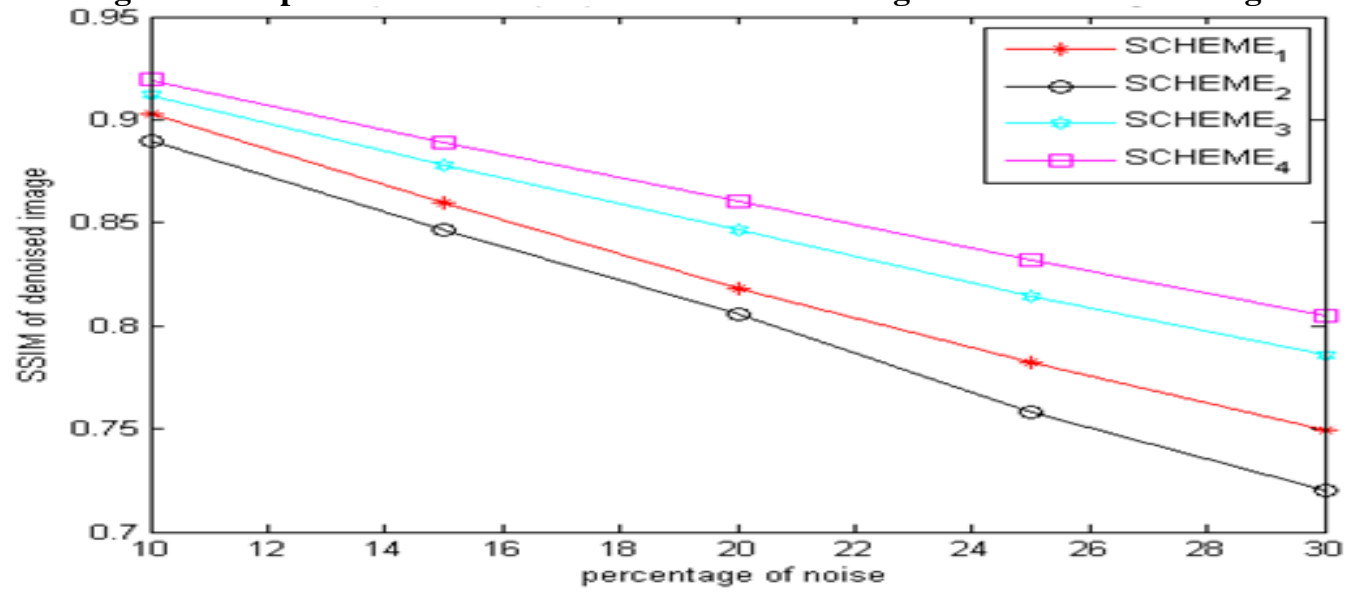

Fig. 6 Comparison of MSSIM Value of four denoising methods for Barbara Image

The most important part of image processing applications is to improve the visual appearance of a digitally captured image. Hence the overall goal is to transform the images in a way that results in sharp and clear images. The visual appearance depends on the visibility of artifacts and the preservation of image edges. Artifacts are due to the modifications of spatial correlation between wavelet coefficients caused by zeroing all neighbouring coefficients. The visual appearance can be seen from the visual images shown in figures 7, 8 and 9 respectively.

The computation time taken by the presented techniques on a computer with Intel core 2 duo CPU @ $2.93 \mathrm{GHz}$ with 2GB RAM for Lena, Boat and Barbara image of size $512 \times 512$ with a standard deviation of $\sigma=[10,15,20,25,30]$ is given in table 3 . In table 3 computation time is better for DWT and SVD based image denoising technique. But it fails in preservation of image details; this can be seen in figures $7 \mathrm{~b}, 8 \mathrm{~b}$ and $9 \mathrm{~b}$. Hence noise removal and preservation of all other details of the image is better only in DTCWT, SVD along with Frobenius correction factor. The graphical analysis of the same is shown in figures 10,11 and 12 respectively.

In addition to the denoising methods, the overall performance depends on the wavelet type and its length. In all denoising techniques discussed here uses a length of six. To avoid boundary distortion symmetric extension is used.

From the computed performance, it can be concluded that 2D-DTCWT incorporated with SVD along with Frobenius energy correction factor and by considering the dependencies between wavelet coefficients using bivariate and bilateral filter provides better PSNR values, visual perception with a reasonable computation time. 


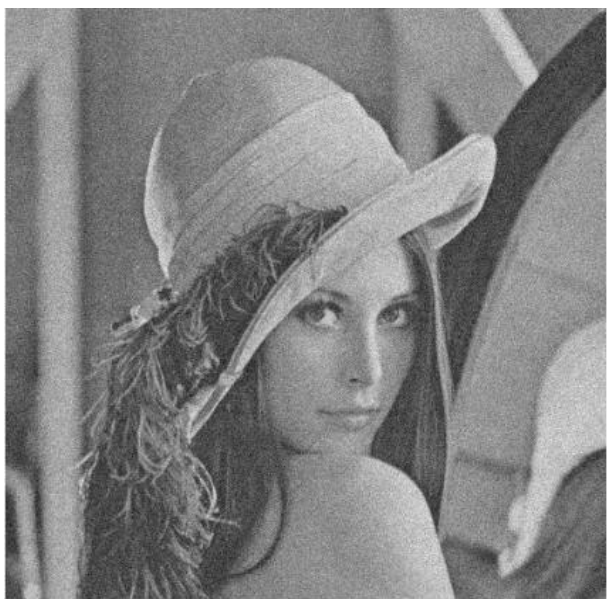

(a)

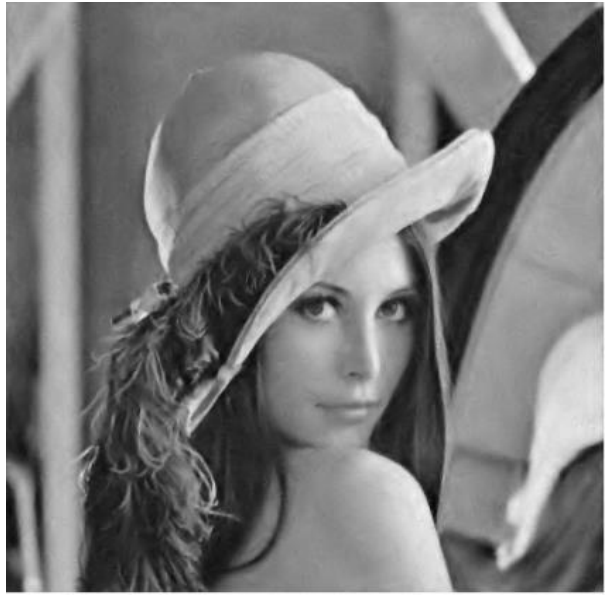

(c)

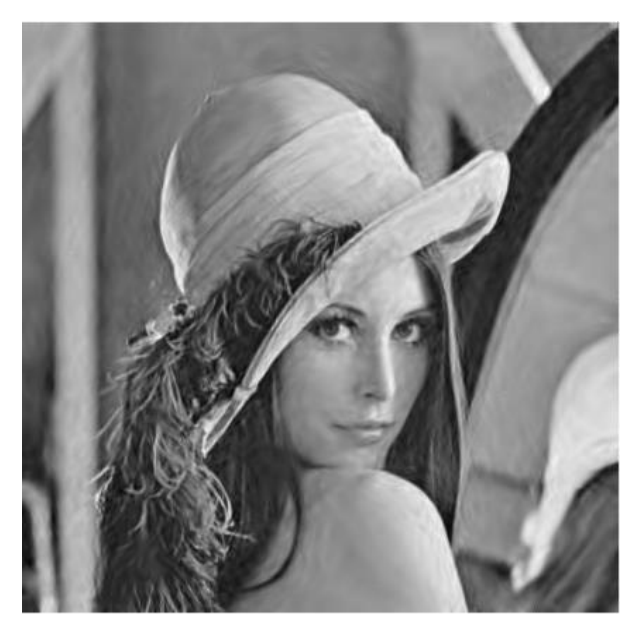

(e)

\section{(e)}

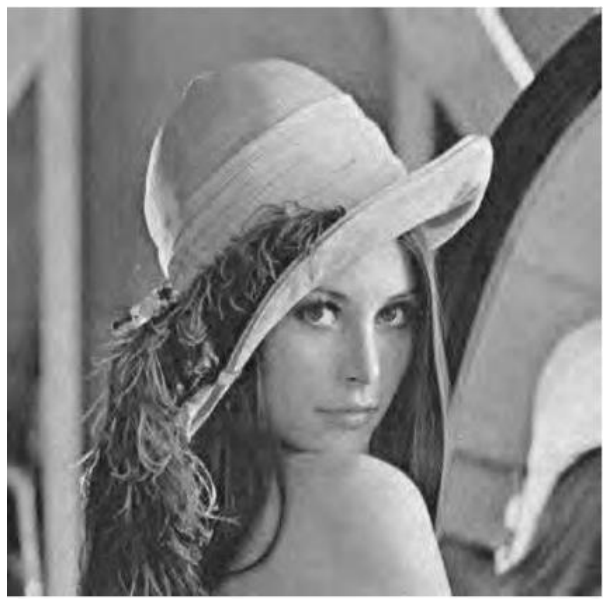

(b)

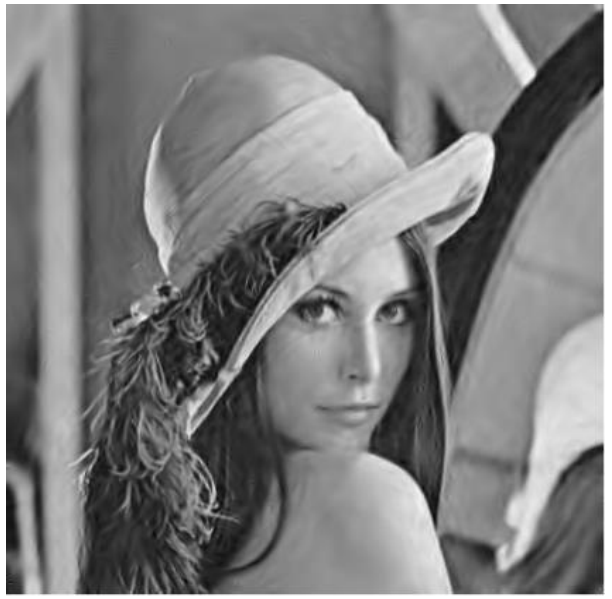

(d)

Denoised image of Lena at noise power level $\sigma=$ (a) Noisy Image, (b) Scheme-1, (c) Scheme-2, (d) Scheme-3, (e) Scheme-4. 


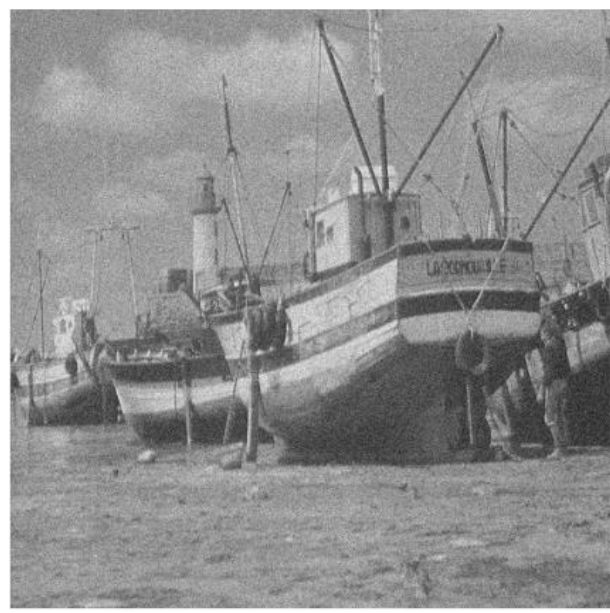

(a)

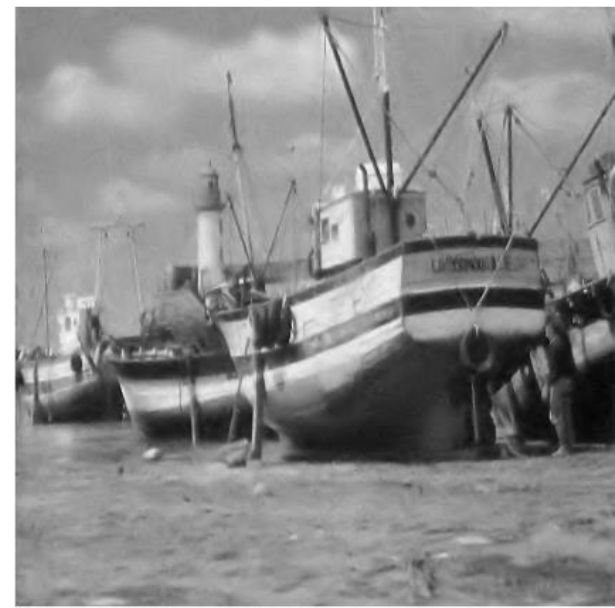

(c)

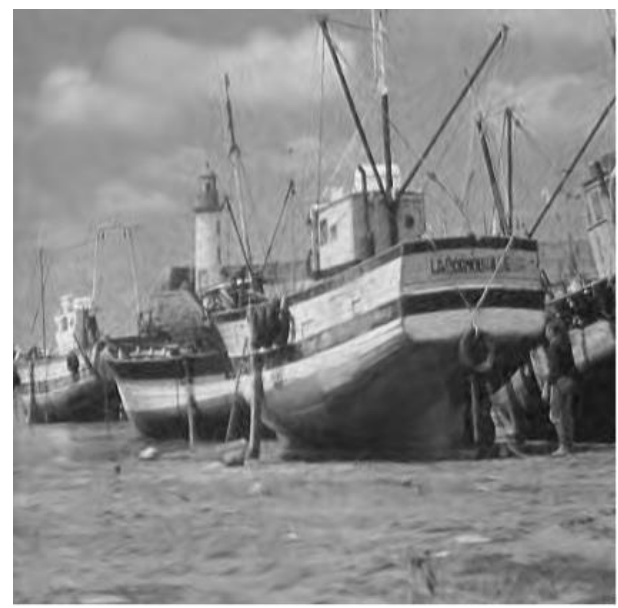

(e)

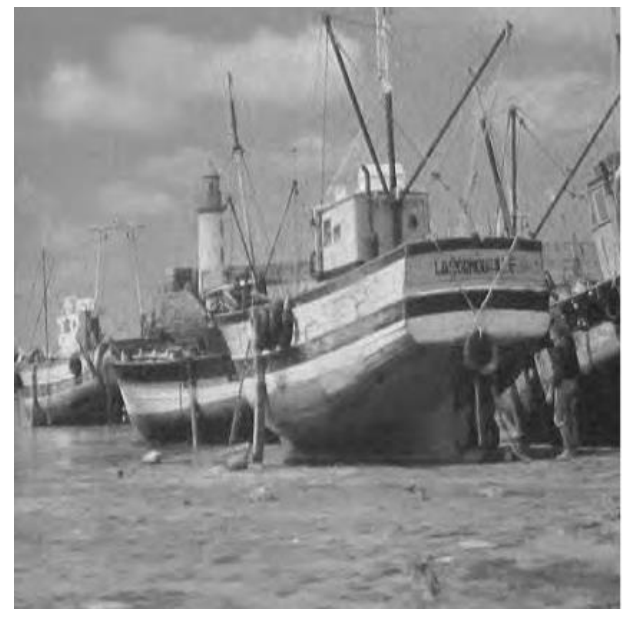

(b)

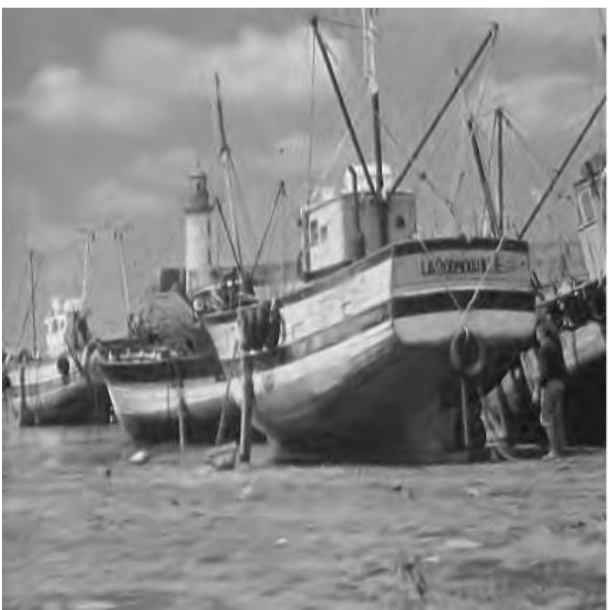

(d) 
Fig. 8

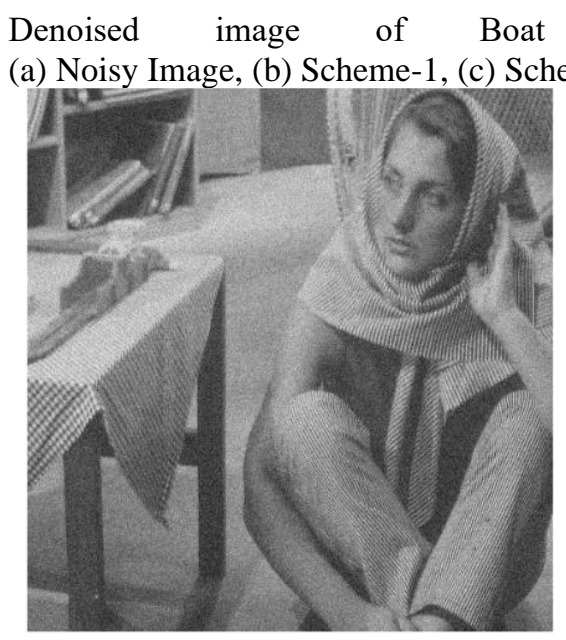

(a)

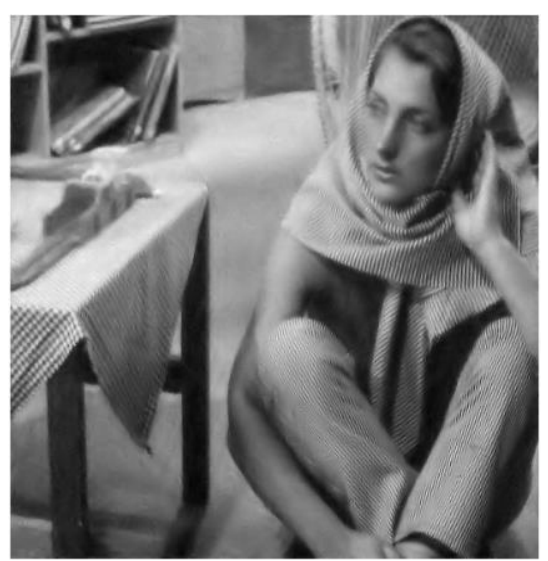

(c) (d)
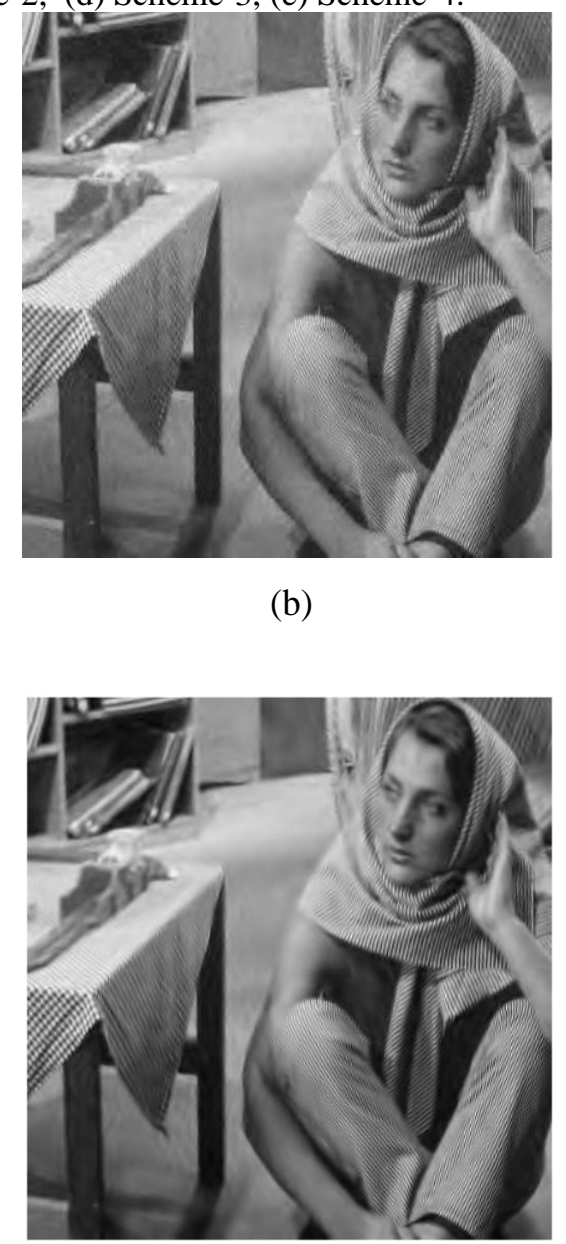

(b)

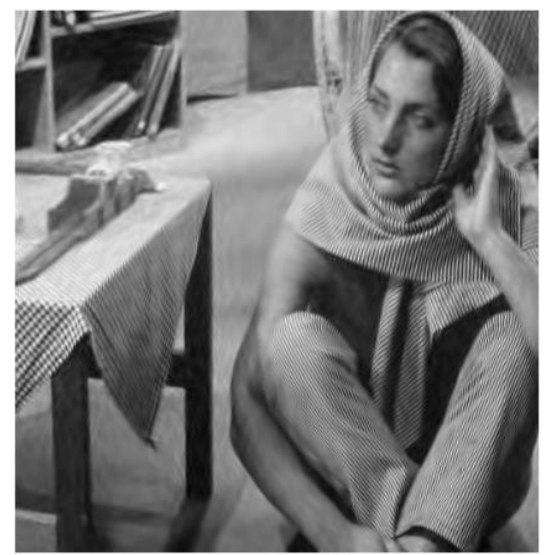

(e)

Fig. 9 Denoised image of Barbara at noise power level $\sigma=20$ (a) Noisy Image, (b) Scheme-1, (c) Scheme-2, (d) Scheme-3, (e) Scheme-4. 
Table 3. Comparison of Time Taken of the four denoising methods

\begin{tabular}{|c|c|c|c|c|}
\hline $\begin{array}{l}\text { Proposed } \\
\text { Methods }\end{array}$ & $\sigma_{\mathbf{n}}$ & LENA & BOAT & BARBARA \\
\hline \multirow{5}{*}{ SCHEME_1 } & 10 & 1.40 & 1.38 & 1.31 \\
\hline & 15 & 1.37 & 1.12 & 1.23 \\
\hline & 20 & 1.31 & 1.18 & 1.37 \\
\hline & 25 & 1.17 & 1.20 & 1.12 \\
\hline & 30 & 1.41 & 1.24 & 1.26 \\
\hline \multirow{5}{*}{ SCHEME_2 } & 10 & 7.90 & 8.25 & 7.81 \\
\hline & 15 & 8.00 & 7.89 & 8.01 \\
\hline & 20 & 8.33 & 8.11 & 8.34 \\
\hline & 25 & 8.70 & 8.54 & 8.64 \\
\hline & 30 & 9.45 & 9.34 & 9.26 \\
\hline \multirow{5}{*}{ SCHEME_3 } & 10 & 2.62 & 2.24 & 2.27 \\
\hline & 15 & 2.37 & 2.12 & 2.12 \\
\hline & 20 & 2.42 & 2.32 & 2.35 \\
\hline & 25 & 2.43 & 2.32 & 2.24 \\
\hline & 30 & 2.44 & 2.29 & 2.34 \\
\hline \multirow{5}{*}{ SCHEME_4 } & 10 & 2.35 & 2.24 & 2.30 \\
\hline & 15 & 2.46 & 2.34 & 2.23 \\
\hline & 20 & 2.43 & 2.29 & 2.43 \\
\hline & 25 & 2.44 & 2.32 & 2.27 \\
\hline & 30 & 2.58 & 2.40 & 2.23 \\
\hline
\end{tabular}

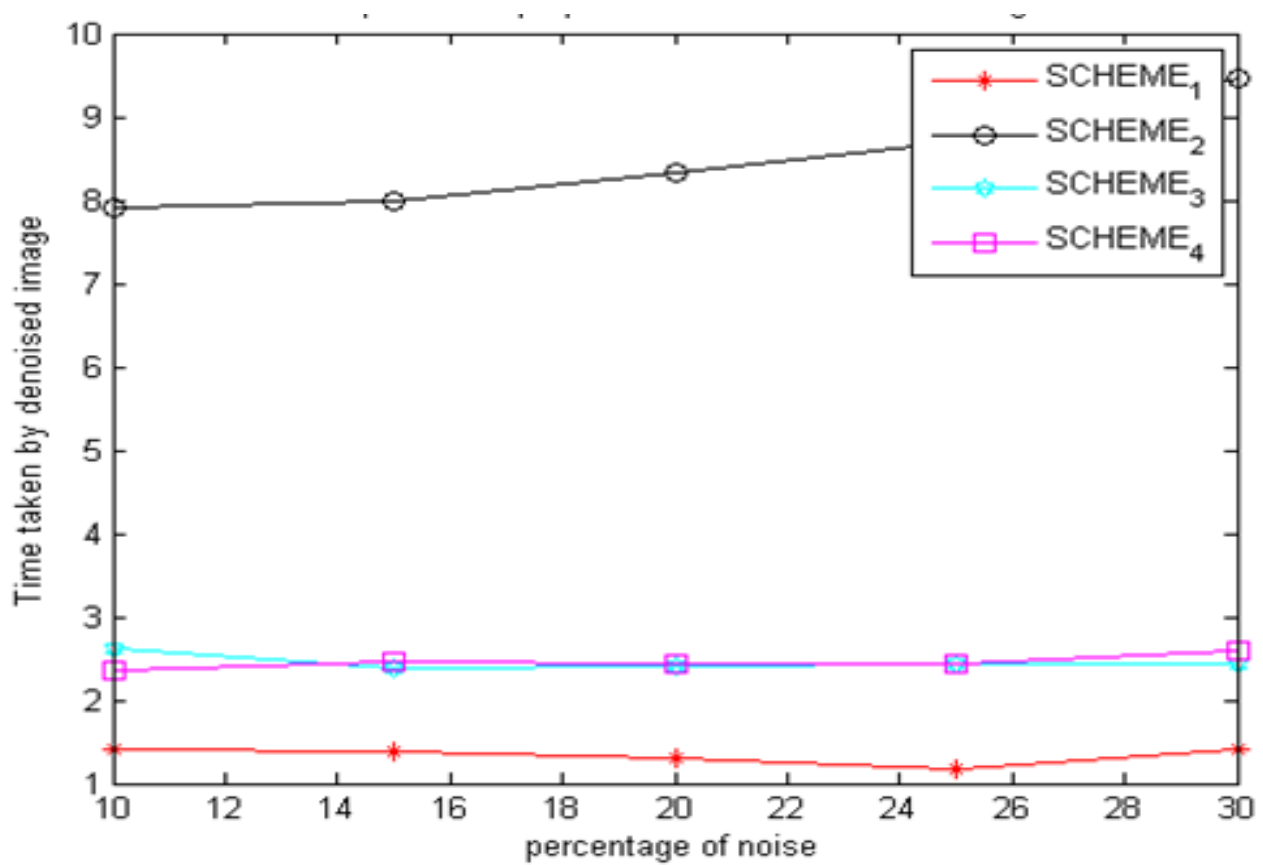

Fig. 10 Comparison of Time of proposed four Methods for Lena Image 


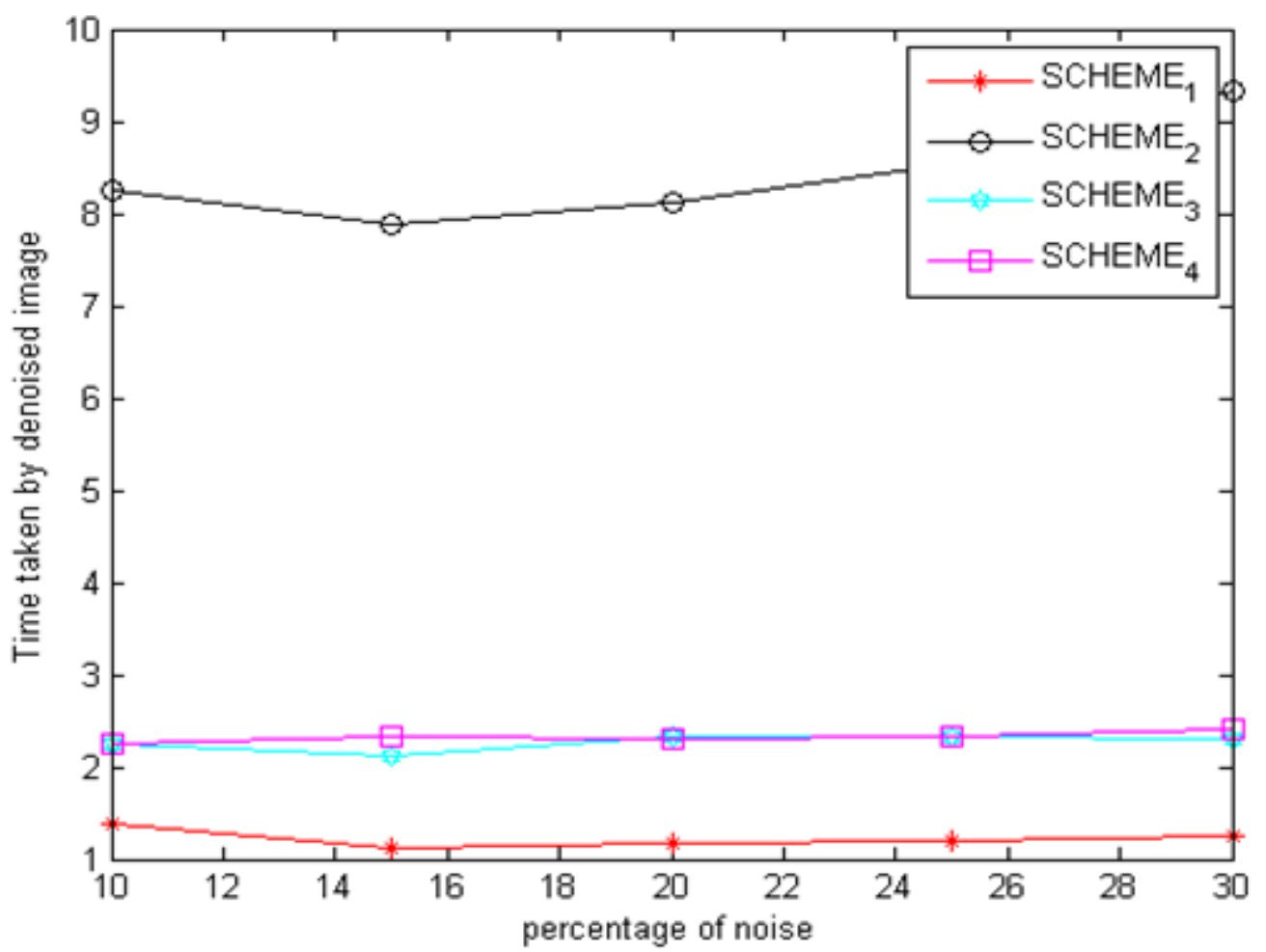

Fig. 11 Comparison of Time of proposed four Methods for Boat Image

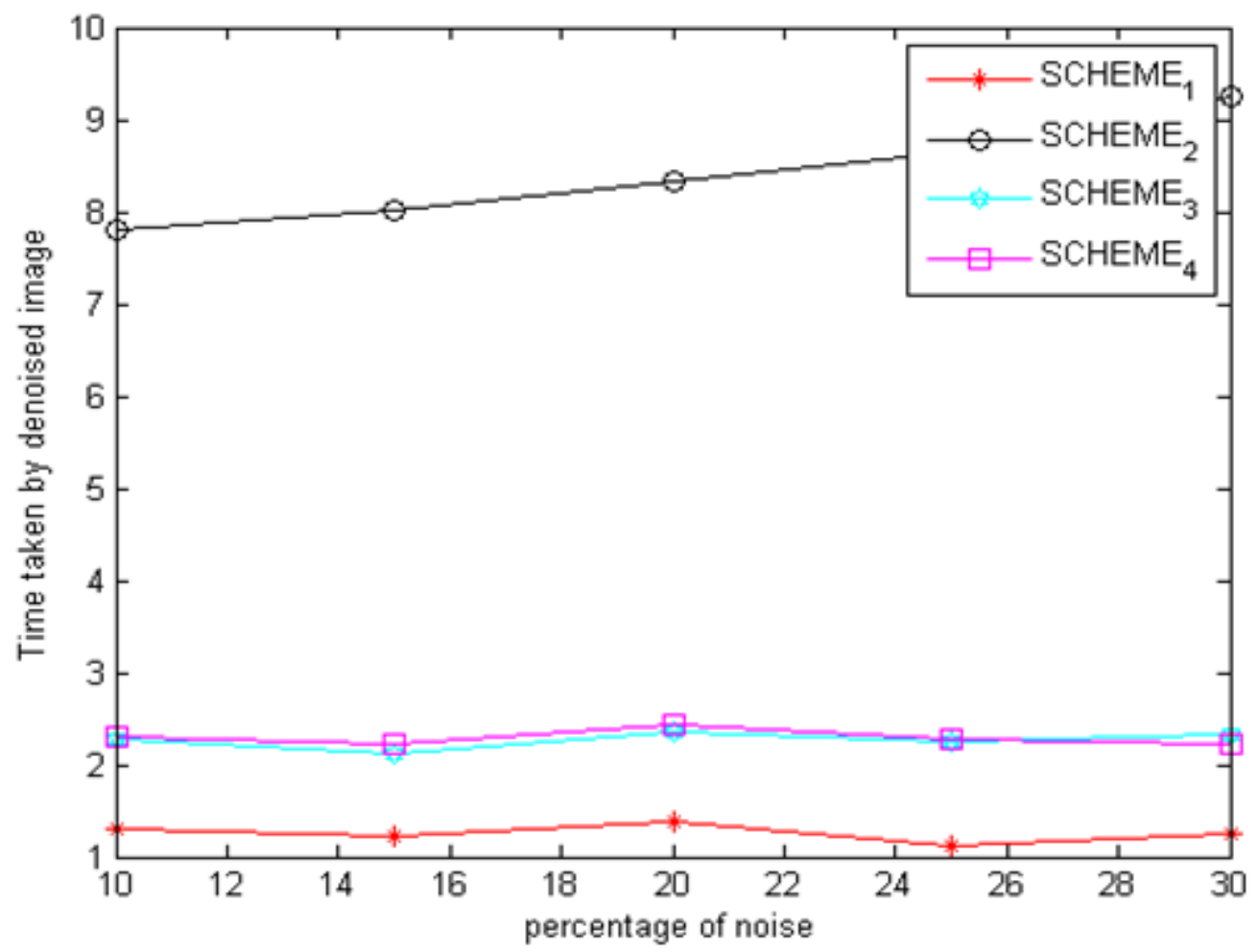

Fig. 12 Comparison of Time of proposed four Methods for Barbara Image 


\section{CONCLUSION}

Image denoising is the major problem in the image processing arena. This is because images are often contaminated by noise during acquisition, transmission and storage. The spatial domains are restricted due to blurring the details of image while removing the noise. However, in transform domain wavelet transform have attained a great success in image processing applications, because wavelet transforms compress the energy of interest into a small number of large coefficients. The limitation of wavelet transform is shift invariance and directional selectivity.

The above said problem is overcome by Dual Tree Complex Wavelet Transform (DTCWT) proposed by Kingsbury. All wavelet based denoising method's performance depends on the threshold value and sparse representation of the image.

In this study four different denoising techniques using different wavelet transforms for images contaminated by AWGN are presented. The first part of the approach is the specific Singular Value Decomposition (SVD) algorithm which approximates the singular values by Frobenius energy correction factor and thresholds the noisy coefficients by considering the wavelet coefficients and their corresponding parent with effective discrete wavelet transform. The second approach is based on real oriented dual tree discrete wavelet transform. NeighSure is used to estimate the threshold value for the whole noisy image instead of sub-bands since computational complexity increases when the decomposition level and image size increases. Further to remove noise in very low frequency and preserve the image details Gaussian bilateral filter is used.

Generally the threshold values are found with the knowledge of noise variance, but the noise variance is not same across the scale. To overcome this sub-band adaptive VisuShrink is derived by using both the signal and noise variance. In the last method DTCWT is used along with SVD. Here the singular values are corrected by a Frobenius energy correction factor. A bivariate shrinkage function is used to remove the noisy information, and finally bilateral filter that uses geometric closeness and gray level value preserves the image details and smooth's the image.

All the presented techniques are within the framework of wavelet transform and provide results that are better than other denoising techniques. The performance improvement can be due to three reasons. One is the sparse representation, which helps to retain the image details, second is the ability to find threshold value by considering inter-scale dependency. Thirdly image specific features like edges are preserved by using non-linear filters.

\section{REFERENCE}

1. Acton, S.T. and Bovik, A.C. (1998), "Nonlinear image estimation using piecewise and local image models", IEEE Transactions on Image Processing, Vol. 7, No. 7, pp. 979-991. https://doi.org/10.1109/83.701153

2. Andrews, H. and Patterson, C. (1976), "Singular value decompositions and digital image processing", IEEE Transactions on Acoustics, Speech, Signal Processing, Vol. 24, No. 1, pp. 26-53. https://doi.org/10.1109/TASSP.1976.1162766

3. Arce, G.R. and Foster, R.E. (1989), "Detailpreserving ranked-order based filters for image processing", IEEE Transactions on Acoustics, Speech, and Signal Processing, Vol. 37, No. 1, pp. 83-98. https://doi.org/10.1109/29.17503

4. Chang, S.G. Yu, B. and Vetterli, M. (2000), "Adaptive wavelet thresholding for image denoising and compression", IEEE Transactions on Image Processing, Vol. 9, No. 9, pp. 15321546. https://doi.org/10.1109/83.862633

5. Chen, G. Zhu, W-P. and Xie, W. (2012), "Wavelet-based image denoising using three scales of dependency", IET Image Processing, Vol. 6, No. 6, pp. 756-760. https://doi.org/10.1049/iet-ipr.2010.0408

6. Chen, G.Y. and Bui, T.D. (2003), "Multiwavelet denoising using neighbouring coefficients", IEEE Signal Processing Letters, Vol. 10, No. 7, pp.211-214.

https://doi.org/10.1109/LSP.2003.811586

7. Chen, G.Y. Bui, T.D. and Krzyzak, A. (2004), "Image denoising using neighbouring wavelet coefficients", Proceedings of IEEE International Conference on Acoustics, Speech, and Signal Processing, Montreal, Quebec, Canada, Vol. 2, pp. 917-920. https://doi.org/10.1109/ICASSP.2004.1326408

8. Cho, D. Bui, T.D. and Chen, G. (2009), "Image denoising based on wavelet shrinkage using neighbor and level dependency", International Journal of Wavelets, Multiresolution and Information Processing, Vol. 7, No. 3, pp. 299311.

https://doi.org/10.1142/S0219691309002945

9. Coifman, R. and Donoho, D.L. (1995), "Timeinvariant wavelet denoising", Proceedings of Wavelet and Statistics, ed. A. Antoniadis and G. Oppenheim, New York: Springer-Verlag, Vol. 
103, pp. 125-150. https://doi.org/10.1007/978-14612-2544-7

10. Dengwen, Z. and Wengang, C. (2008, "Image denoising with an optimal threshold and neighbouring window", Pattern Recognition Letters-Elsevier, Vol. 29, No. 11, pp. 1694-1697. https://doi.org/10.1016/j.patrec.2008.04.014

11. Donoho, D.L. (1995), "Denoising by softthresholding", IEEE Transactions on Information Theory, Vol. 41, No. 3, pp. 613-627. https://doi.org/10.1109/18.382009

12. Donoho, D.L. and Johnstone, I.M. (1994), "Ideal spatial adaptation by wavelet shrinkage", Journal of Biometrika, Vol. 81, No. 3, pp. 425455. https://doi.org/10.1093/biomet/81.3.425

13. Donoho, D.L. Johnstone, I.M. Kerkyacharian, G. and Picard, D. (1995), "Wavelet shrinkage: Asymptopia?", Journal of Royal Statistical Society-Series B, Vol. 57, No. 2, pp. 301-369. https://doi.org/10.1111/j.25176161.1995.tb02032.x

14. Faruk, SK.U, Ramanaiah, K.V. and Soundararajan, K. (2017), "FPGA Implementation of Image Denoiser using Dual Tree Complex Wavelet Transform", International Journal of Applied Engineering Research, Vol. 12, No. 14, pp. 4083-4089. https://www.ripublication.com/ijaer17/ijaerv12 n14 06.pdf

15. Gabbouj, M. Coyle, E.J. and Gallagher, N.C. (1992), "An overview of median and stack filtering", Journal of Circuits, Systems, and Signal Processing, Vol. 11, No. 1, pp.7-45. https://doi.org/10.1007/BF01189220

16. Hari, O. and Mantosh, B. (2012), “An improved image denoising method based on wavelet thresholding", Journal of Signal and Information Processing, Vol. 3, No. 1, pp. 109116. https://doi.org/10.4236/jsip.2012.31014

17. Hari, O. and Mantosh, B. (2015), “A generalized image denoising method using neighbouring wavelet coefficients", Journal of Signal, Image and Video Processing-Springer, Vol. 9, No. 1, pp. 191-200. https://doi.org/ 10.1007/s11760013-0434-5

18. Iqbal M.Z. Ghafoor, A. Siddiqui, A.M. Riaz, M.M. and Khalid, U. (2014), "Dual-tree complex wavelet transform and SVD based medical image resolution enhancement", Journal of Signal Processing-Elsevier, Vol. 105, pp. 430-447.

https://doi.org/10.1016/j.sigpro.2014.05.011

19. Kakarala, R. and Ogunbona, P.O. (2001), "Signal analysis using a multiresolution form of the singular value decomposition", IEEE
Transactions on Image Processing, Vol.10, No.5, pp. 724-735. https://doi.org/10.1109/83.918566

20. Khare, A. Tiwary, U.S. Pedrycz, W. and Jeon, M. (2010), "Multilevel adaptive thresholding and shrinkage technique for denoising using Daubechies complex wavelet transform", The Imaging Science Journal, Vol. 58, No. 6, pp. 340-358.

https://doi.org/10.1179/136821910X127503391 $\underline{75826}$

21. Kingsbury, N.G. (1998), "The dual-tree complex wavelet transform: A new technique for shift invariance and directional filters", Proceedings of 8th IEEE DSP Workshop, Utah, No.86, pp. 912.

https://pdfs.semanticscholar.org/294e/f14804ee b9bed629eab9ff974ca3d0ffe92a.pdf

22. Kingsbury, N.G. (1999), "Image processing with complex wavelets", Philosophical Transactions of the Royal Society, Vol. 357, No. 1760, pp. 2543-2560.

https://doi.org/10.1098/rsta.1999.0447

23. Kingsbury, N.G.( 2001), "Complex wavelets for shift invariant analysis and filtering of signals", Applied and Computational Harmonic Analysis, Vol. 10, No. 3, pp. 234-253. https://doi.org/10.1006/acha.2000.0343

24. Laavanya, M. and Karthikeyan, M. (2016), "Singular value decomposition using bivariate model in wavelet domain for image denoising", International Journal of Printing, Packaging and Allied Sciences, Vol. 4, No. 3, pp. 17841791.

25. Laavanya, M. and Karthikeyan, M. (2017), "Real oriented dual tree wavelet transform with an optimal threshold using neighbor coefficients and Gaussian bilateral filter for image denoising", Asian Journal of Research in Social Sciences and Humanities, Vol. 7, No. 3, pp. 1070-1079. https://doi.org/10.5958/22497315.2017.00228.3

26. Laavanya, M. and Karthikeyan, M. (2018), "Dual tree complex wavelet transform incorporating SVD and bilateral filter for image denoising", International Journal of Biomedical Engineering and Technology (Inderscience), Vol. 26, No. 3, pp. 266-278. https://doi.org/10.1504/IJBET.2018.089956

27. Laavanya, M. and Vijayaraghavan, V. (2019), "A Sub-Band Adaptive Visushrink in Wavelet Domain for Image Denoising", International

28. Lavanya, P.V. Narasimhulu, C.V. and Satya Prasad, K. (2020), "Dual Stage Bayesian Network with Dual-Tree Complex Wavelet 
Transformation for Image Denoising", Journal of Engineering Research, Vol. 8, No. 1, pp. 154178. https://doi.org/10.36909/jer.v8i1.6043

29. Lee, Y. and Kassam, S. (1985), "Generalized median filtering and related nonlinear filtering techniques", IEEE Transactions on Acoustics, Speech, and Signal Processing, Vol. 33, No. 3, pp. 672-683. https://doi.org/10.1109/TASSP.1985.1164591

30. Mallat, S.G. (1989), "A theory for multiresolution signal decomposition: The wavelet representation", IEEE Transactions on Pattern Analysis and Machine Intelligence, Vol.11, No. 7, pp. 674-693. https://doi.org/10.1109/34.192463

31. Mohideen, K.S. Perumal, A.S. and Sathik, M.M. (2008), "Image de-noising using discrete wavelet transform", International Journal of Computer Science and Network Security, Vol.8, No.1, pp. 213-216. http://paper.ijcsns.org/07_book/200801/200801 29.pdf

32. Naveed, K. Shaukat, B. Ehsan, S. McdonaldMaier, K.D. and Rehman, N. (2019), "Multiscale image denoising using goodness-of-fit test based on EDF statistics", PLoS ONE, Vol. 14, No. 5, pp. $1-25$. https://doi.org/10.1371/journal.pone.0216197

33. Ozkan, M.K. Erdem, A.T. Sezan, M.I. and Tekalp, A.M. (1992), "Efficient multiframe Wiener restoration of blurred and noisy image sequences", IEEE Transactions on Image Processing, Vol. 1, No. 4, pp. 453-476. https://doi.org/10.1109/83.199916

34. Raghuveer, R.M. and Bopardikar, A.S. (1999), "Wavelet transforms: Introduction to theory and application", Pearson Education, Asia.

35. Remenyi, N. Nicolis, O. Nason, G. and Vidakovic, B. (2014), "Image denoising with 2D scale-mixing complex wavelet transforms", IEEE Transactions on Image Processing, Vol. 23, No. 12, pp. 5165-5174. https://doi.org/10.1109/TIP.2014.2362058

36. Selesnick, I.W. Baraniuk, R.G. and Kingsbury, N.G. (2005), "The dual-tree complex wavelet transform", IEEE Signal Processing Magazine, Vol. 22, No. 6, pp. 123-151. https://doi.org/10.1109/MSP.2005.1550194

37. Sendur, L. and Selesnick, I.W. (2002a), "Bivariate shrinkage functions for waveletbased denoising exploiting interscale dependency", IEEE Transactions on Signal Processing, Vol. 50, No. 11, pp. 2744-2756. https://doi.org/10.1109/TSP.2002.804091
38. Sendur, L. and Selesnick, I.W. (2002b), "Bivariate shrinkage with local variance estimation", IEEE Signal Processing Letters, Vol. 9, No. 12, pp. 438-441. https://doi.org/10.1109/LSP.2002.806054

39. Shreyamshakumar, B.K. (2013), "Image denoising based on Gaussian/bilateral filter and its method noise thresholding", Proceedings of Signal, Image and Video Processing-Springer, Vol. 7, No. 6, pp. 1159-1172. https://doi.org/10.1007/s11760-012-0372-7

40. Smith, S.M. and Brady, J.M. (1997), "SUSANA new approach to low level image processing", International Journal of Computer Vision, Vol. 23, No. 1, pp. 45-78. https://doi.org/10.1023/A:1007963824710

41. Tomasi, C. and Manduchi, R. (1998), "Bilateral filtering for gray and color images", Proceedings of Sixth IEEE International Conference on Computer Vision, Bombay, India, pp. 839-846. https://doi.org/10.1109/ICCV.1998.710815

42. Wongsawat, Y. Rao, K.R. and Oraintara, S. (2005), "Multichannel SVD-based image denoising", IEEE International Symposium on Circuits and Systems, Vol. 6, pp. 5990-5993. https://doi.org/10.1109/ISCAS.2005.1466004

43. Yu, X. Liang, W. Zhang, L. Jin, H. and Qiu, J. (2016), "Dual-tree complex wavelet transform and SVD based acoustic noise reduction and its application in leak detection for natural gas pipeline", Journal of Mechanical Systems and Signal Processing-Elsevier, Vol.72-73, pp. 266285. https://doi.org/10.1016/j.ymssp.2015.10.034

44. Zhang, M. and Gunturk, B.K. (2008), "Multiresolution bilateral filtering for image denoising", IEEE Transactions on Image Processing, Vol. 17, No. 12, pp. 2324-2333. https://doi.org/10.1109/TIP.2008.2006658

45. Zhang, Y-X. Chen, L. and Jia, J. (2013), “Image de-noising using an improved bivariate threshold function in Tetrolet domain", Proceedings of International Conference on Image Processing, Computer Vision, and Pattern Recognition, Las Vegas Nevada, USA, Vol. 1, pp. 551-556. https://pdfs.semanticscholar.org/80ca/94cd19ef 7fffa8e3ed511504fd8122f2e68e.pdf

46. Zujun, H. (2003), "Adaptive singular value decomposition in wavelet domain for image denoising", Journal of Pattern RecognitionElsevier, Vol. 36, No. 8, pp. 1747-1763. https://doi.org/ 10.1016/S0031-3203(02)00323$\underline{0}$ 